\title{
Traceable Sea Water Temperature measurements performed by Optical fibers
}

C. García Izquierdo $^{1}$, A. Garcia-Benadí ${ }^{2}$, P. Corredera ${ }^{3}$, S. Hernandez ${ }^{1}$, A. Gonzalez Calvo $^{1}$, J. del Río Fernandez ${ }^{2}$, M. Nogueres-Cervera ${ }^{2}$, C. Pulido de Torres ${ }^{4}$, D. del Campo $^{1}$

${ }^{1}$ Centro Español de Metrología, CEM, Tres Cantos, Madrid, SPAIN, mcgarciaiz@cem.minetur.es

${ }^{2}$ Research group SARTI UPC,Vilanova I la Geltrú, SPAIN, albert.garciabenadi@upc.edu

${ }^{3}$ Instituto de Óptica, IO - CSIC, Madrid, SPAIN, p.corredera@csic.es

${ }^{4}$ Instituto de Estructura de la Materia, CSIC, Madrid, SPAIN, conchi.pulido@io.cfmac.csic.es

\begin{abstract}
This paper describes a technique to perform traceable temperature measurements of the seawater column and seawater surface, based on optical fiber Bragg gratings. The paper explains the different phases of the work done: design of the optical fibers, its optical and thermal calibration and onsite measurements of the seawater temperature. In the design of these thermometers, special attention was paid to the involved materials in order to prevent any damage due to the exposure to such rugged environment.

The fibers were subjected to optical and thermal calibration, with the aim to get traceable measurements and reliable uncertainty calculation of the seawater temperature. The fibers were deployed in the Mediterranean sea and water temperatures were continuously monitored and compared with the most common used thermometer in these environment, CTD, located in the submarine observatory OBSEA.
\end{abstract}

This paper is part of a Special Issue entitled VSI: 2017 IMEKO TC 19.

\section{Research Highlights}

- A technique, based on fiber Bragg gratings (FBG), to measure traceable seawater temperature was developed

- Design, characterization together with the optical and thermal calibration of the FBG was performed

- Onsite seawater temperature measurements and its continuous monitoring by using FBG were performed in the Mediterranean submarine observatory OBSEA

\section{Abbreviations}

CTD, device to measure conductivity, temperature of the seawater and depth; FBG, Fiber Bragg Grating; OBSEA, underwater observatory (www.obsea.es); Pt-100, platinum resistance thermometer with a value of $100 \Omega$ at the triple point of water; Pt-25, platinum resistance thermometer with a value of $25 \Omega$ at the triple point of water

\section{Keywords}

Metrology, Uncertainty, Sea surface temperature, Sea profile temperature, Fiber Optic, Fiber Bragg grating. 


\section{Introduction}

Understanding the behaviour, evolution and characteristics of the sea is an essential issue to address some of the raging global concerns of the moment: climate change, the carbon cycle, ocean acidification, variations in the marine circulation and their effects, etc. For this reason, the number of studies about the sea has been increasing in recent years.

The quantity sea temperature is explicitly mentioned in Annex III of the Marine Strategy Framework Directive of the European Union [1] as one of the parameters requiring attention. Meaningful and coherent measurements of seawater temperature are essential to understand its influence in nearly all marine processes, as well as to understand the heat exchange mechanisms between the sea and the atmosphere.

Currently, seawater temperature is mainly measured by three different methods. In the first one, the temperature measurements are performed via satellite observation. A second method consists on the attachment of thermometers to buoys and their deployment into the sea, at fixed positions. In the third one, the seawater temperature is measured in research campaigns, by ships, where different arrays, CTDs, are launched in order to perform measurements of conductivity, temperature, and depth. Each method present disadvantages: temperature measurements by satellites are not possible at higher sea depths than 1 meter, the buoys provide measurements at fixed sea depths and the research campaigns do not supply measurements for long periods.

The CTD is the most acceptable instrument by within the scientific community for marine measurements. The measurements of seawater temperature profile imply manual intervention like the deployment of a CTD in a profiler. The CTD is deployed from a vessel with a winch from the sea surface to the seabed in a specific point and time. The CTD records temperature and other magnitudes in its internal memory. Other techniques uses underwater vehicles like the Argo floats that moves free thanks to the water currents, equipped with a CTD performing vertical profiles in a 10 days interval and then through a satellite link the data is available on open repositories after data quality control procedures. Both solutions (manual deployment of using a float) provide water temperature profile in a delayed mode and without a permanent position.

Two different options allow the monitoring of water temperature profile in a specific position and for the long term. One option is the use of a permanent winch on a surface buoy or at the seabed, moving periodically a CTD up and down and other option is to deploy an array of thermistors coupled on a mooring line with an inductive modem to provide real time measurements.

Drawbacks for such systems could be the power consumptions for the winch system and the number of measurements points for the thermistor array.

The presented work is indented to explore a new technique, based on fiber optics, that could improve the problems of the actual systems in terms of power consumption and number of measurements points on the water columns. The use of thermometers based on the distribution of temperature sensors along an optical fibre is proposed, explained and analysed. This type of thermometer allows to measure seawater temperature profile, seawater surface temperature and to monitor both variables during long periods of time.

The paper is structured in seven sections. In the first one, the design and optical calibration of the fiber optics, performed at IO-CSIC, is described. Then the thermal calibration of 
the optical fibers and CTD, performed by CEM, is analysed. The fifth section explains the deployment of the fiber optics in the permanent underwater observatory OBSEA [2] performed by UPC. The sixth section describes the on site measurements of seawater temperature profile and sea surface temperature. The last section compares the temperature measurements taken by the fiber optics and by the CTDs.

\section{Design and optical characterization of the fiber optics.}

The use of fiber optics as thermometers presents the advantages of its immunity to electromagnetic interferences, its multiplexing capacity, reliable work in hostile environments and that they can play the role of both, sensor and transmission medium, in a unique device and at the same time. Other advantage is the fiber optics do not generate any impact in their surrounding due to their passive nature, small size and low weight.

A measurement instrument, based on fiber optics, is composed by three principal elements: the sensor, the transmission medium and an optoelectronic device, known as interrogator. The sensor is the element whose properties, in the guiding of the light, depends on the physical magnitude of interest. The detection of the magnitude change by the sensor can be based on different physical phenomena and in linear or non linear properties of the optical fiber. The choosing of the most suitable physical phenomena, and for hence, the most appropriate sensor depends on the intended use.

The optical measurement instrument used in this experiment consists on the use of Bragg grating working in reflexion mode. When the interrogator illuminates, with a broadband spectrum light, the fiber optic, each Bragg grating reflects the light pulse, whose specific wavelength depends on the Bragg grating period. The rest of the light is transmitted without any variation (figure 1). A change of temperature produces a small variation in the Bragg gratings' period and, for hence; it generates changes in the wavelength of the reflected light, to be detected by the interrogator (figure 2).

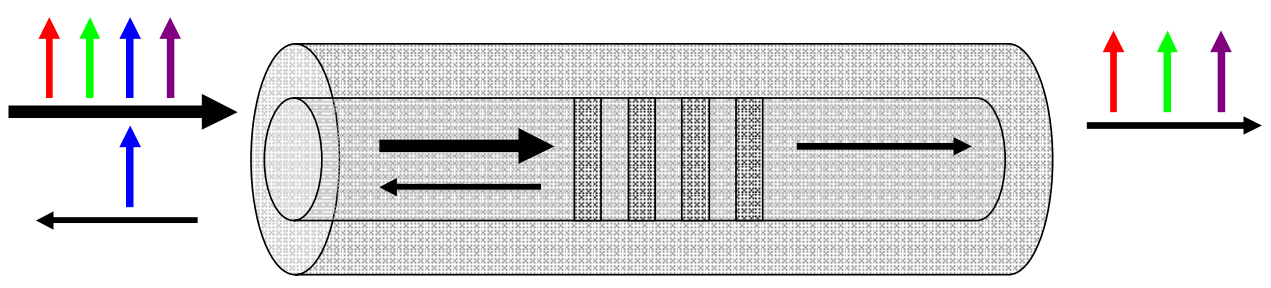

Figure 1. Theoretical explanation of the FBG working principle. 

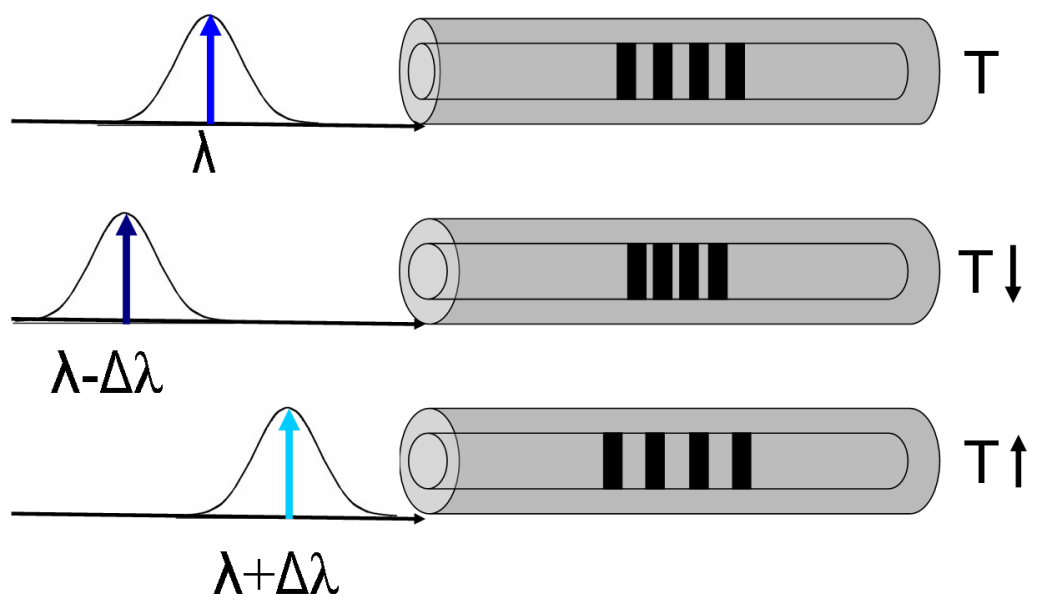

Figure 2. Theoretical explanation about the use of FBG as thermometers

The multiplexing capacity means that several Bragg gratings can be recoded in a unique fiber. In this experiment, two different fiber optics were designed, one of them with ten measurement points and the other one with three measurement points, both distributed in $40 \mathrm{~m}$ optical fiber. The reason of the two designs is to keep the 10 points fiber, the „measurement fiber", permanently in the seawater, since its extraction and deployment in the sea is a risk of its damage. The fiber optic with three points of measurements, called the "control fiber", would work as a drift control of the measurement fiber. However, at the end, both fibers were taken from the sea to check their drift by their calibration at the beginning and at the end of the experiment.

The FBGs designed for this experiment were written on Single Mode Optical Fiber SM-ITU652 coated with Acrylate. In the design of these fiber optics, special attention was payed to the involved materials in order to prevent damage in such rugged environment. The fiber is inside a 1/4" x 0.35 wall thickness $316 \mathrm{~L}$ stainless steel tube and the final encapsulation is done with a layer of Polypropylene/PEEK resistant to seawater.

After the design and assembly of the optical fibers, they were calibrated. The optical calibration of the FBG implies the determination of the measurement errors of the interrogator, MicronOptics ${ }^{\circledR}$ model SM125. The calibration was performed according the procedure and technique developed at IO-CSIC [3]. Figure 3 shows the optical calibration, where the uncertainty evaluation includes all the influence quantities. The uncertainty calculation was performed by following the requirements expressed in the Guide to the expression of uncertainty in measurements guide, GUM [4]. 


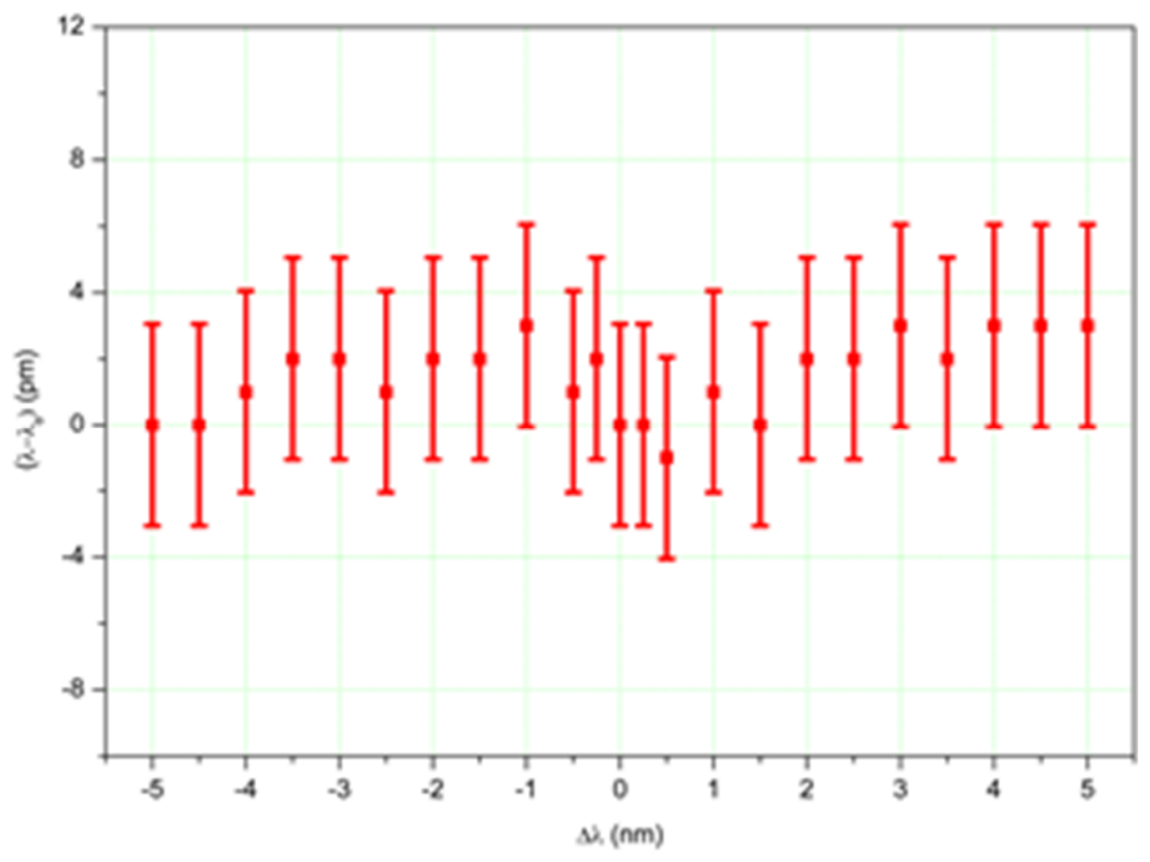

Figure 3. SM-125 calibration results

\section{Calibration of the fiber optics as thermometers.}

\subsection{Determination of the wavelength displacement with temperature}

The wavelength displacement with the change of temperature was determined by the thermal calibration of the optical system: fiber optics and interrogator, by using the CEM Spanish national temperature standards. The calibration was performed in the temperature range $(0,30)^{\circ} \mathrm{C}$ in a 195 litre calibration bath, specifically designed for this purpose. The results of the calibrations of the two optical fibers are shown in figures 4 and 5.

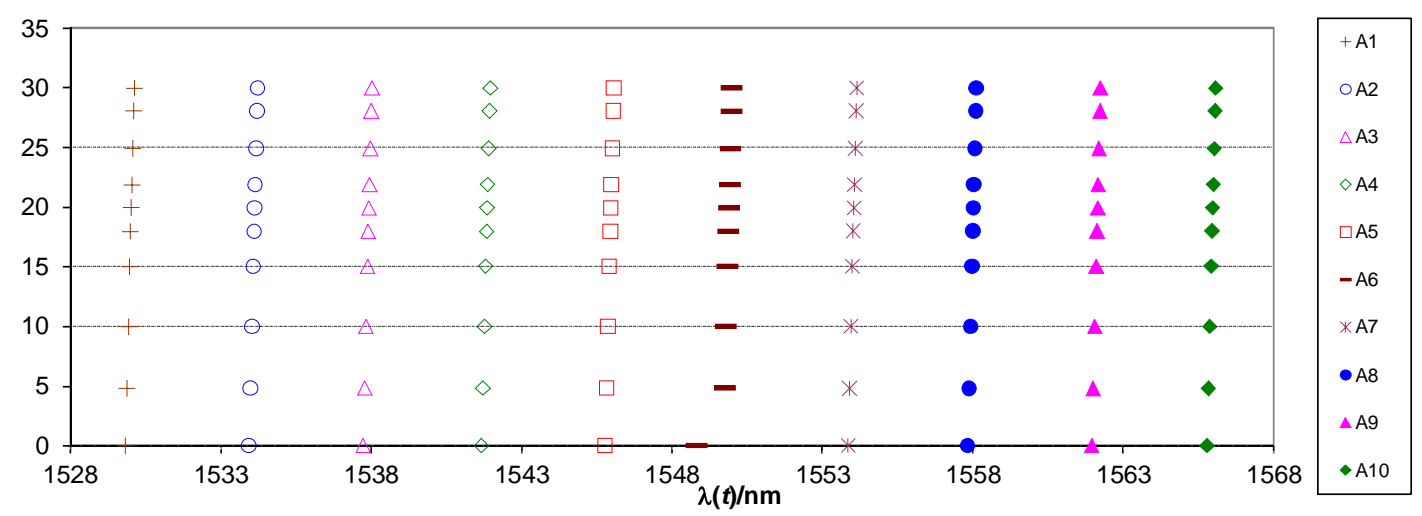

Figure 4. Thermal calibration of the optical fiber, with 10 measurements points. 


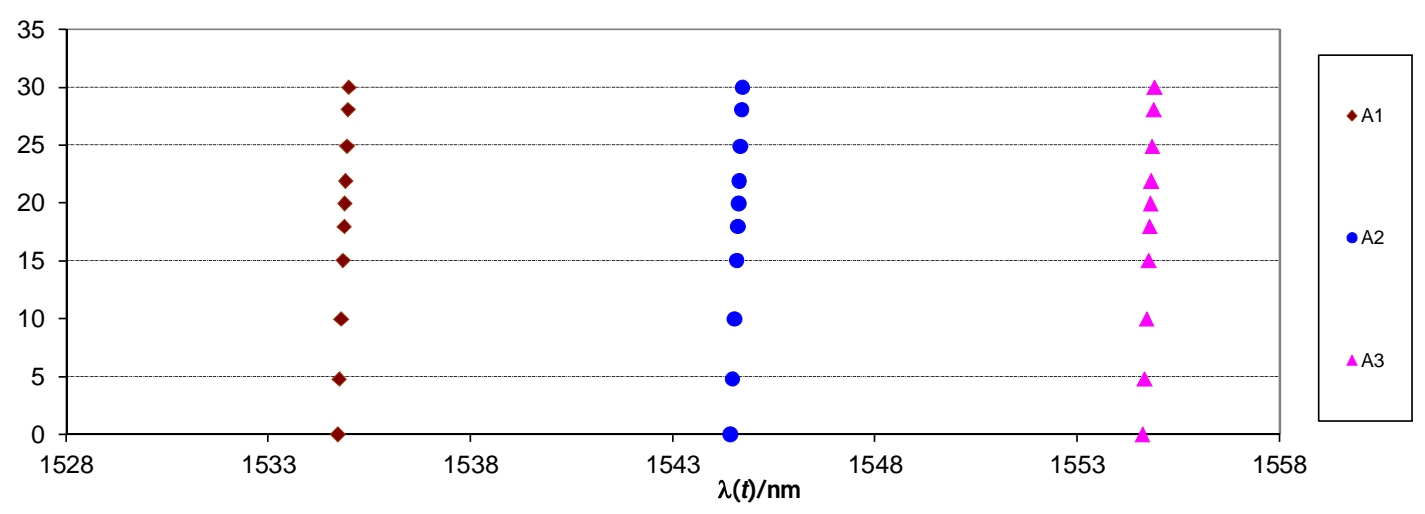

Figure 5. Thermal calibration of the optical fiber, with 3 measurements points.

The mathematical model of the thermal calibration of FBG at each temperature is expressed by the equation (1)

$$
\lambda(t)=\lambda\left(t_{r e f}\right)+s_{t}\left(t-t_{r e f}\right)
$$

Where:

- $\lambda(t)$ is the value of the wavelength at the temperature $t$.

- $\lambda\left(t_{r e f}\right)$ is the wavelength at the reference temperature $t_{r e f .}$

- $s_{t}$ is the sensitivity coefficient of each temperature sensor inside the optical fiber at the temperature $t$, expressed in $\mathrm{nm} /{ }^{\circ} \mathrm{C}$.

- $\quad t$ is the real temperature of the isothermal enclosure.

- $t_{\text {ref }}$ is the reference temperature.

The reference temperature is determined by the average of the readings of two platinum resistance thermometers, Pt-100, calibrated by comparison with an expanded uncertainty $(k=2)$ of $10 \mathrm{mK}$. The associated mathematical model of the reference temperature is expressed in equation 2 .

$$
t_{\text {ref }}=\frac{1}{2}\left(t_{1}+\delta t_{1 \mathrm{c}}+\delta t_{1 \mathrm{~d}}+t_{2}+\delta t_{2 \mathrm{c}}+\delta t_{2 \mathrm{~d}}\right)+\delta t_{\text {stability }}+\delta t_{\text {uniformity }}
$$

The value of the reference temperature, determined by the standard thermometers is corrected by factors related with the corresponding calibration certificates, $\delta t_{\text {ic }}$, drifts, $\delta t_{\text {id }}$ and the influences of the calibration bath, its stability and its uniformity, $\delta t_{\text {stability, }}$ $\delta t_{\text {uniformity. }}$. The stability and uniformity of the calibration bath was determined at each calibration point by the two standard thermometers, having a maximum value of $9 \mathrm{mK}$ for both components.

The temperature determination by the standard thermometers is performed by their electrical resistance values, $R_{\mathrm{i}}\left(t_{r e f}\right)$ measured with a resistance bridge, ASL, F-700. The resistance values are corrected by the influences of the bridge and the standard resistor, following the mathematical model of equation 3.

$$
R_{\mathrm{i}}\left(t_{r e f}\right)=\left(L_{x}+\delta L\right) \times\left(R_{\mathrm{s}}+\delta R_{\mathrm{sd}}\right)
$$

Where: 
- $\quad L_{x}$ is the resistance ratio of the standard thermometer and standard resistor, read by the resistance bridge

- $R_{\mathrm{S}}$ is the standard resistor

- $\quad \delta R_{\text {sd: }}$ drift of the standard resistor $R_{\mathrm{s}}$

- $\delta L$ : indeterminacy of the bridge reading due to its own uncertainty

In general, the quantities with the symbol ' $\delta$ ' in equations (2) and (3) can be estimated as zero, but they have associated uncertainties.

The relationship between temperature and wavelength was determined in the calibration range by the calibration curve. Figures 4 and 5 show how the radiation wavelength, reflected by each Bragg grating, increases linearly with temperature. The calibration curve was calculated by using least-squares straight-line fit of the wavelengths at different temperatures (equation 4).

$$
t=a \cdot \lambda+t_{0}
$$

The calibration curves were calculated in the range $(5,30){ }^{\circ} \mathrm{C}$. This range was not extended to $0{ }^{\circ} \mathrm{C}$ because the very high residuals at this points meaning a nonlinear behaviour of the fibers in the temperature range of $(0,5){ }^{\circ} \mathrm{C}$. Further investigation is needed in this matter.

The calibration of the optical fibers showed that their sensitivity in temperture, $s_{t}$, in this range is $0.1^{\circ} \mathrm{C} / \mathrm{pm}$.

\subsection{Uncertainty calculation}

The uncertainty associated at each temperature sensor inside the fiber optics and at each temperature is calculated by applying the law of propagation of uncertainties [4] to (1), considering all the variables in (1) not correlated.

$$
u^{2}(\lambda(t))=u^{2}\left(\lambda\left(t_{r e f}\right)\right)+s_{x}{ }^{2} u^{2}\left(t_{\text {ref }}\right)
$$

Where:

- $\quad u\left(\lambda\left(t_{r e f}\right)\right)$ : is the uncertainty due to the wavelength reading by the interrogator.

- $u\left(t_{r e f}\right):$ is the uncertainty of the calibration bath temperature at each of the measurement points.

The contribution represented by $u\left(t_{r e f}\right)$ can be shifted into its different components and by applying the law of propagation of uncertainties to (2), in the following way:

$u^{2}\left(t_{\text {ref }}\right)=\frac{1}{4}\left[u\left(\delta t_{1 \mathrm{c}}\right)+u\left(\delta t_{2 \mathrm{c}}\right)\right]^{2}+\frac{1}{4} \llbracket\left[u^{2}\left(t_{1}\right)+u^{2}\left(t_{2}\right)+u^{2}\left(\delta t_{1 \mathrm{~d}}\right)+u^{2}\left(\delta t_{2 \mathrm{~d}}\right)\right]+u^{2}\left(\delta t_{\text {stability }}\right)+u^{2}\left(\delta t_{\text {uniformity }}\right)$

The standard thermometers were previously calibrated at the same time, with the same equipment, against the same reference standards and with the same procedure. Then, $\delta t_{1 \mathrm{c}}$ y $\delta t_{2 \mathrm{c}}$ are correlated, with a correlated coefficient very close to 1 . We consider the value of this coefficient is 1 , as the less favourable case. 
The two standards are of the same model, so the equation (6) can be simplified with the assumption that $u\left(t_{1}\right)=u\left(t_{2}\right)=u\left(t_{\mathrm{s}}\right)$ y $u\left(\delta t_{1 \mathrm{c}}\right)=u\left(\delta t_{2 \mathrm{c}}\right)=u\left(\delta t_{\mathrm{sc}}\right)$ :

$$
u^{2}\left(t_{\text {ref }}\right)=u^{2}\left(\delta t_{\mathrm{sc}}\right)+\frac{1}{4}\left[2 \cdot u^{2}\left(t_{s}\right)+u^{2}\left(\delta t_{1 \mathrm{~d}}\right)+u^{2}\left(\delta t_{2 \mathrm{~d}}\right)\right]+u^{2}\left(\delta t_{\text {stability }}\right)+u^{2}\left(\delta t_{\text {uniformity }}\right)
$$

And considering that $t_{\mathrm{s}}$ is derived from the reading of the electrical resistance of the two standard thermometers in the resistance bridge, (3):

$$
u^{2}\left(t_{s}\right)=\left(\frac{R_{\mathrm{s}}}{s_{\mathrm{p}}}\right)^{2}\left[u^{2}\left(L_{\mathrm{p}}\right)+u^{2}(\delta L)\right]+\left(\frac{L_{\mathrm{p}}}{s_{\mathrm{p}}}\right)^{2}
$$

Then, the uncertainty associated to the determination of the reference temperature is:

$$
\begin{gathered}
u^{2}\left(t_{\text {ref }}\right)=\frac{1}{2}\left(\frac{R_{\mathrm{s}}}{s_{\mathrm{p}}}\right)^{2}\left[u^{2}\left(L_{\mathrm{p}}\right)+u^{2}(\delta L)\right]+\frac{1}{2}\left(\frac{L_{\mathrm{p}}}{s_{\mathrm{p}}}\right)^{2}\left[u^{2}\left(R_{\mathrm{s}}\right)+u^{2}\left(\delta R_{\mathrm{sd}}\right)\right]+u^{2}\left(\delta t_{\mathrm{sc}}\right)+ \\
+\frac{1}{4}\left[u^{2}\left(\delta t_{1 \mathrm{~d}}\right)+u^{2}\left(\delta t_{2 \mathrm{~d}}\right)\right]+u^{2}\left(\delta t_{\text {stability }}\right)+u^{2}\left(\delta t_{\text {uniformity }}\right)
\end{gathered}
$$

\begin{tabular}{|c|c|c|c|c|c|c|}
\hline $\begin{array}{l}\text { Source of } \\
\text { uncertainty }\end{array}$ & Units & & $\begin{array}{l}\text { probability } \\
\text { distribution }\end{array}$ & Divisor & $\begin{array}{l}\text { coefficient } \\
\mathrm{ci}=\partial f / \partial x i\end{array}$ & $\begin{array}{c}\text { Standard } \\
\text { uncertainty } \\
\text { component, } \\
u(x i) / \stackrel{\circ}{C}\end{array}$ \\
\hline \multicolumn{7}{|l|}{ Laboratory system } \\
\hline$L p$ & $\Omega / \Omega$ & 0.000001 & Rectangular & $\sqrt{3}$ & $R \mathrm{~s} / \mathrm{sp}$ & 0.00015 \\
\hline$d L$ & $\Omega / \Omega$ & 0.0000061 & Rectangular & $\sqrt{3}$ & $R s / s p$ & 0.00090 \\
\hline Rs & $\Omega$ & 0.000021 & Normal & 2 & Lref/sp & 0.00005 \\
\hline $\mathrm{d} R \mathrm{sd}$ & $\Omega$ & 0.0001 & Rectangular & $\sqrt{3}$ & Lref/sp & 0.00030 \\
\hline$\delta t c$ & $\stackrel{\circ}{ } \mathrm{C}$ & 0.01 & Normal & 2 & 1 & 0.00500 \\
\hline$\delta t d$ & $\stackrel{\circ}{ } \mathrm{C}$ & 0.005 & Rectangular & $\sqrt{3}$ & 1 & 0.00289 \\
\hline$\delta$ te & $\stackrel{\circ}{ } \mathrm{C}$ & 0.009 & Rectangular & $\sqrt{3}$ & 1 & 0.00520 \\
\hline$\delta t \mathrm{u}$ & $\stackrel{\circ}{ } \mathrm{C}$ & 0.009 & Rectangular & $\sqrt{3}$ & 1 & 0.00520 \\
\hline \multicolumn{7}{|c|}{ Characteristics of the fiber optic: } \\
\hline$\delta \lambda /$ resolution & $\mathrm{pm}$ & 0.5 & Rectangular & $\sqrt{3}$ & $1 / s_{t}$ & 0.03 \\
\hline
\end{tabular}

Table 1 summarises the complete calibration uncertainty budget, with the all uncertainty component and the corresponding probability distribution. The total expanded uncertainty $(k=2)$ for the calibration of the FBGs as thermometers is $60 \mathrm{mK}$.

\begin{tabular}{|ll}
\hline Combined uncertainty/ ${ }^{\circ} \mathrm{C}$ & \\
Expanded Uncertainty/ $\stackrel{\circ}{\mathrm{C}} \quad k=2$ & 0.03 \\
\hline
\end{tabular}

Table 1. Calibration uncertainty budget for the FBG as thermometers. 
The uncertainty associated to the calibration function (4) could be calculated in a simplified form as the combination of the calibration uncertainty at each temperature (5) and the standard deviation of the fit residuals, as it is expressed in the equation (10).

$$
U(t)=2 \cdot \sqrt{\left(\left(\frac{U_{\text {calibration }}}{2}\right)^{2}+s_{\text {residuals }}^{2}\right)}
$$

\subsection{Drift of the fiber optics.}

The fiber optics were calibrated just before their deployment and after three months working under the sea. Both calibrations were performed with the same procedure and the same uncertainty calculation already explained. The drift of the fiber optics was calculated as the difference between the two calibrations. Figure 6 and 7 show the drift of the Bragg gratings for the fiber optic with ten and three measurement points respectively.

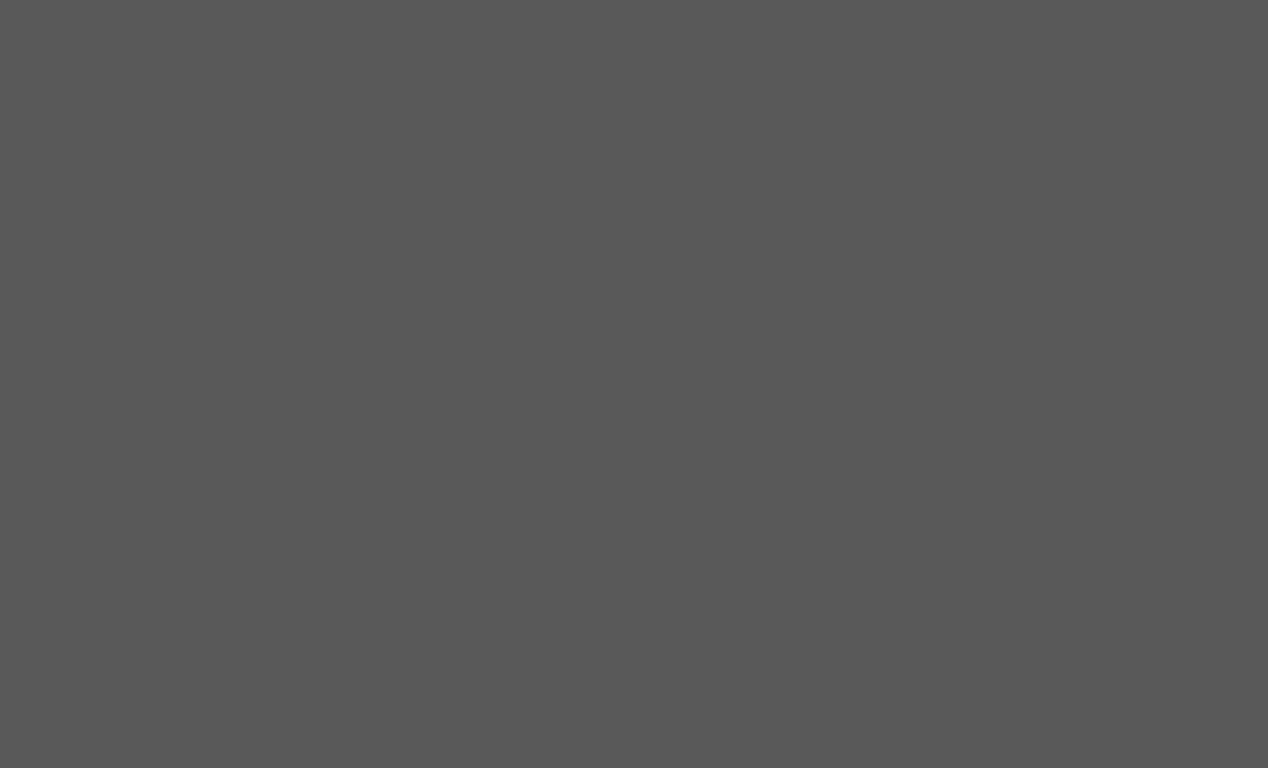

Figure 6. Drift of the ten points FBG after three months under the sea.

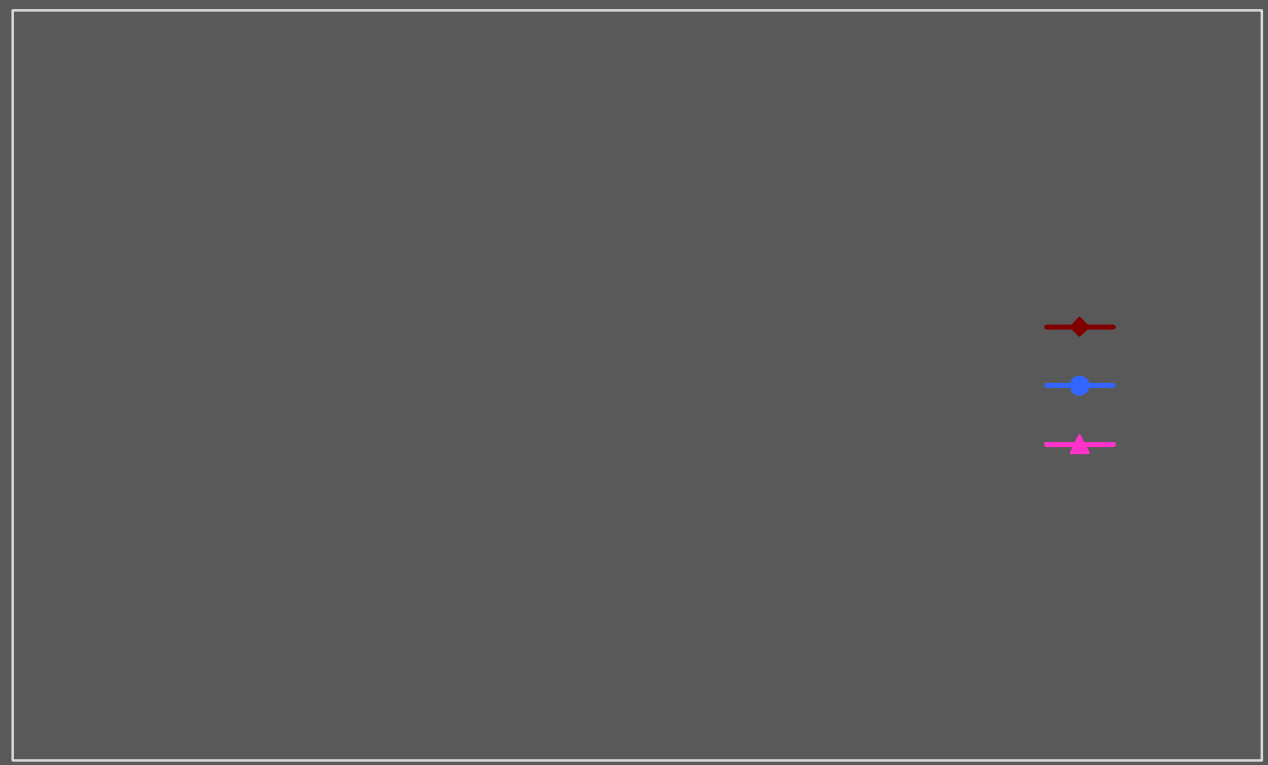

Figure 7. Drift of the three points FBG after three months under the sea. 
Figure 6 shows that the drift of almost all Bragg gratings are of the same order as the resolution of the fiber optic, being the drift almost covered by the calibration uncertainty. Higher drift is shown in the first Bragg grating, located outside the seawater level. The $6^{\text {th }}$ Bragg grating, located at $0.5 \mathrm{~m}$ under the water, was severe damaged during its exposure at saline environment. The same happened to the $2^{\text {nd }}$ Bragg grating of the fiber optic with three points of measurements, located also at $0.5 \mathrm{~m}$ under the water. The others Bragg gratings suffered a drift lower than $0.2^{\circ} \mathrm{C}$ (Figure 7).

\section{Thermal calibration of CTD.}

\subsection{Determination of the correction}

The seawater temperature is usually measured by devices, known as CTDs that also perform measurements of the seawater conductivity and the depth of the CTD position. In the seabed of the submarine observatory OBSEA, there is a CTD, whose temperature measurements can be compared with the temperature measurements of fiber optics. Reliable comparison is only possible if the CTD is thermally calibrated. The calibration of CTD was performed in the calibration bath described previously to which an equalizer block was added in order to improve its thermal stability and uniformity, having values of $2 \mathrm{mK}$ and $1 \mathrm{mK}$ respectively.

The mathematical model of the CTD calibration follows the equation (11)

$C=t_{\text {ref }}-t_{C T D}$

Where:

- $\quad C$ is the correction of the CTD temperature reading

- $t_{\text {ref }}$ is the reference temperature.

- $t_{C T D}$ is the CTD's temperature reading

The CTD was calibrated in the range $(0,30){ }^{\circ} \mathrm{C}$ and the reference temperature at each calibration point was determined by two resistance thermometers, Pt-25, previously calibrated in the fixed points of the International Temperature Scale of 1990 with an expanded uncertainty $(k=2)$ of $2.2 \mathrm{mK}$. The mathematical model of the reference temperature is expressed in the equation (7) and, as in the case of the calibration of the optical fibers, the value of the electrical resistance $R_{i}\left(t_{r e f}\right)$ of the Pt-25 at each temperature is measured by a resistance bridge, ASL, F-700, (equation 3).

The submarine observatory OBSEA works with the CTD, Seabird model 37SMP. The calibration results with the corresponding uncertainty calculation is shown in figure 8 and table 2. Figure 8 shows the calibration uncertainty at $5^{\circ} \mathrm{C}$ that is higher than the uncertainty at the other temperatures because of the worst performance in stability and uniformity of the calibration bath. 


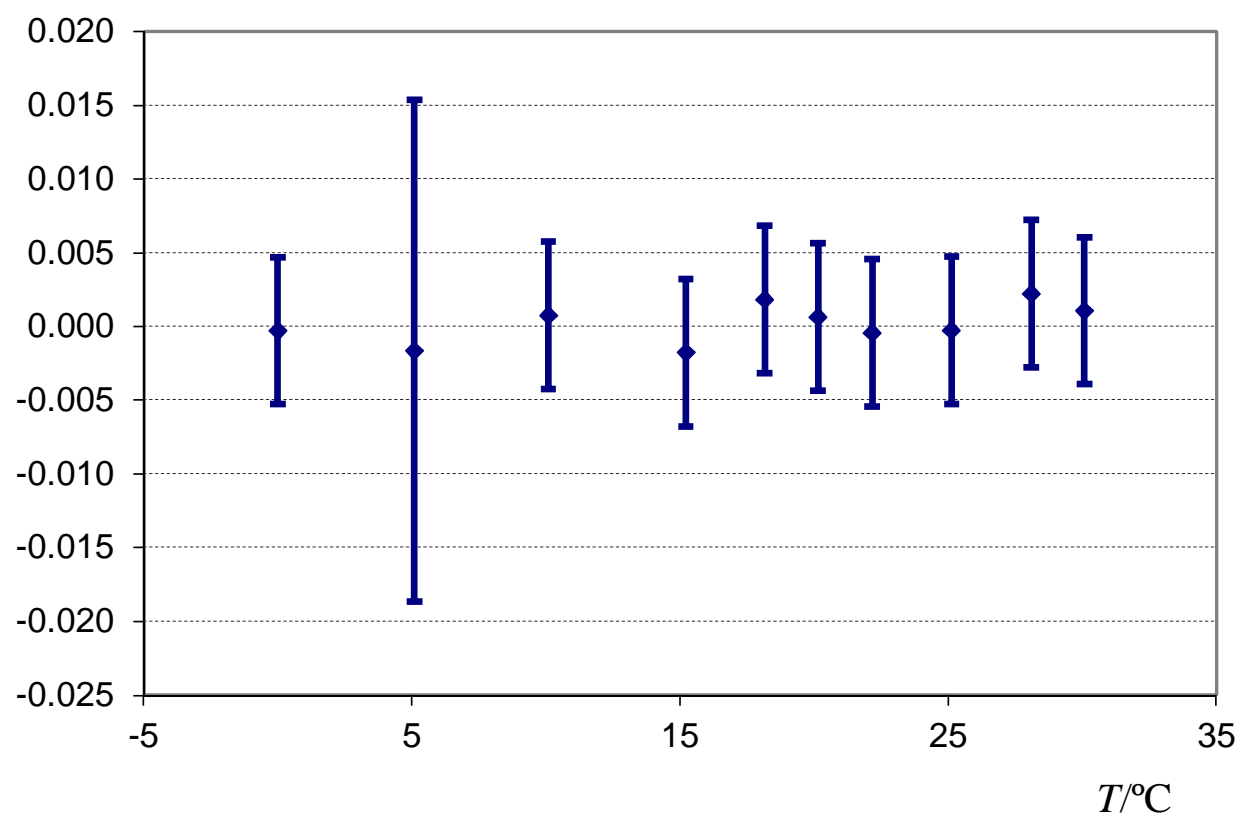

Figure 8. Thermal calibration of the CTD seabird 37SMP

\subsection{Uncertainty calculation}

The uncertainty associated with the correction of the CTD is calculated by applying the law of propagation of uncertainties [4] to (11), considering that all the variables in (11) are not correlated.

$$
u^{2}(C)=u^{2}\left(t_{r e f}\right)+u^{2}\left(t_{C T D}\right)
$$

Where the associated uncertainty to the reference temperature, $u^{2}\left(t_{r e f}\right)$ is calculated by the equation 9.

Table 2 shows the complete calibration uncertainty budget, with all uncertainty components and their corresponding probability distribution. The total expanded uncertainty $(k=2)$ for the thermal calibration of the CTD is $5 \mathrm{mK}$.

\begin{tabular}{|c|c|c|c|c|c|c|}
\hline $\begin{array}{c}\text { Quantity } \\
x_{i}\end{array}$ & Unit & Uncertainty & $\begin{array}{l}\text { Probability } \\
\text { distribution }\end{array}$ & Divisor & $\begin{array}{c}\text { Sensitivity } \\
\text { coefficient } \\
C_{i}\end{array}$ & $\begin{array}{c}\text { Standard } \\
\text { deviation } \\
u\left(x_{i}\right) \\
{ }^{\circ} \mathrm{C}\end{array}$ \\
\hline \multicolumn{7}{|c|}{ Measuring system of the laboratory } \\
\hline$L_{p}$ & $\Omega / \Omega$ & $1.00 \mathrm{E}-06$ & Rectangular & $\sqrt{3}$ & $R_{\mathrm{s}} / \mathrm{sp}$ & $1.48 \mathrm{E}-04$ \\
\hline$\delta L$ & $\Omega / \Omega$ & $6.10 \mathrm{E}-06$ & Rectangular & $\sqrt{3}$ & $R_{\mathrm{s}} / \mathrm{sp}$ & 9.03E-04 \\
\hline$R_{\mathrm{s}}$ & $\Omega$ & $2.80 \mathrm{E}-04$ & Normal & 2 & Lret/sp & $7.18 \mathrm{E}-04$ \\
\hline$\delta R_{\mathrm{sd}}$ & $\Omega$ & $1.00 \mathrm{E}-04$ & Rectangular & $\sqrt{3}$ & Lref/sp & $2.96 \mathrm{E}-04$ \\
\hline$\delta t_{c}$ & ${ }^{\circ} \mathrm{C}$ & 0,0022 & Normal & 2 & "'1"' & $1.00 \mathrm{E}-03$ \\
\hline
\end{tabular}




\begin{tabular}{|c|c|c|c|c|c|c|}
\hline$\delta t_{\mathrm{d}}$ & ${ }^{\circ} \mathrm{C}$ & 0.002 & Rectangular & $\sqrt{3}$ & 1 & $1.15 \mathrm{E}-03$ \\
\hline$\delta t_{e}$ & ${ }^{\circ} \mathrm{C}$ & 0.002 & Rectangular & $\sqrt{3}$ & 1 & $1.15 \mathrm{E}-03$ \\
\hline$\delta t_{\mathrm{u}}$ & ${ }^{\circ} \mathrm{C}$ & 0.001 & Rectangular & $\sqrt{3}$ & 1 & $5.77 \mathrm{E}-04$ \\
\hline \multicolumn{7}{|c|}{ Characteristics of the CTDs } \\
\hline$t_{i}$ & ${ }^{\circ} \mathrm{C}$ & 0.0005 & Rectangular & $\sqrt{3}$ & 1 & 0.0003 \\
\hline & & \multicolumn{2}{|c|}{$\begin{array}{l}\text { Combined uncertainty } \\
\text { Expanded uncertainty }\end{array}$} & $\begin{array}{l}{ }^{\circ} \mathrm{C} \\
\circ\end{array}$ & $k=2$ & 0.002 \\
\hline
\end{tabular}

Table 2. Uncertainty budget of the calibration of CTD.

\subsection{Drift of the CTD}

The drift of the CTD was calculated by the difference between the calibration corrections got in the calibrations before and after the onsite measurements in the sea. The drift of the CTD seabird 37SMP is lower than $3 \mathrm{mK}$ in the calibration range as it is shown in figure 9.

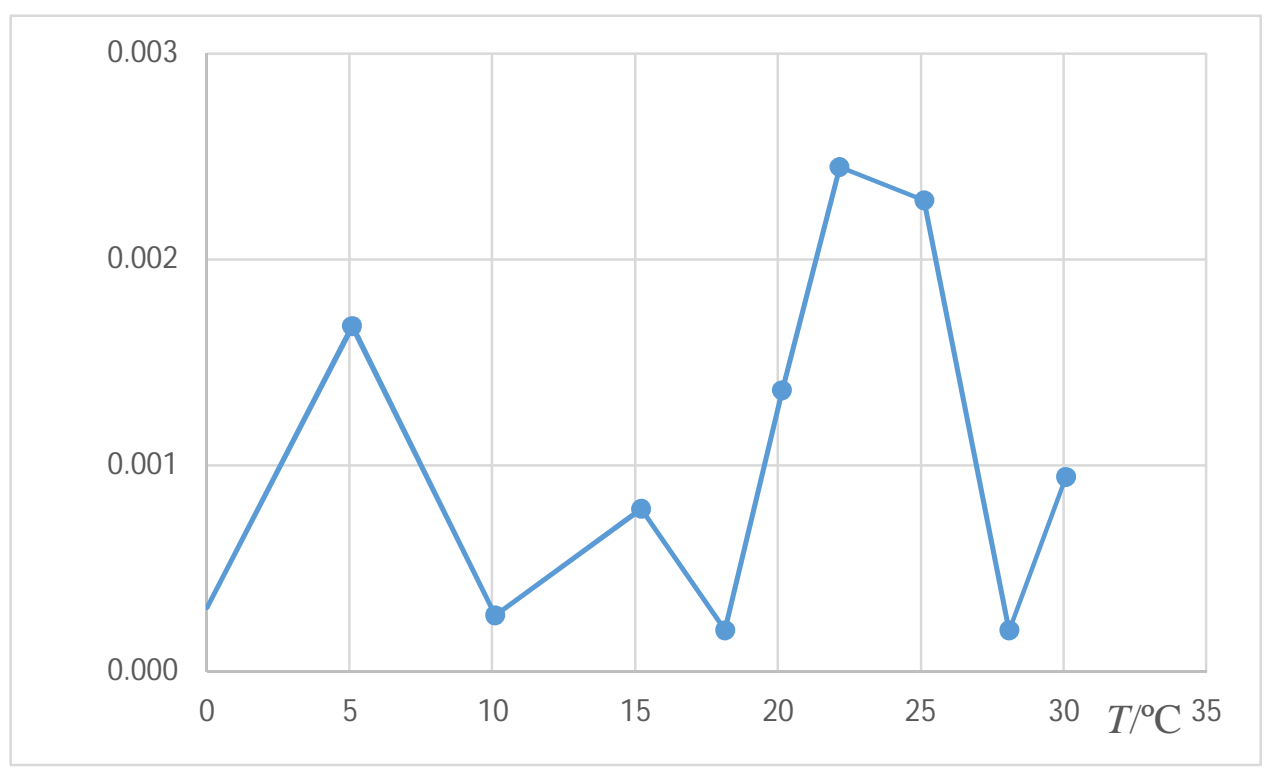

Figure 9. Drift of the CTD seabird 37SMP

\section{Deployment of the fiber optics under the seawater}

The fiber optics were deployed, following the draft of figure 10, between a buoy in the sea surface and the OBSEA underwater observatory, located on the seabed, at $20 \mathrm{~m}$ sea depth, close to the coast of Vilanova i la Geltrú (Barcelona, Spain).

The interrogator of the fiber optics was located on the seabed. As this system is not water proof, a holder for keeping the interrogator underwater was designed and built. Several pressure tests in a hyperbaric chamber were performed in order to guaranty the lack of contact of the interrogator with the sea water. This watertight container for the fiber optic interrogator has been built as a cylinder of 1 meter long and a diameter of $400 \mathrm{~mm}$ made of stainless steel AISI316L. Inside the cylinder has been installed the interrogator MicronOptics $®$ model sm125, a power supply, an Ethernet switch and a microcontroller device with a temperature and humidity sensor. The most critical point of the watertight 
container were the cable glands for the fiber optic ingress. For this purpose metallic AGRO Ex cable glands with additional protection of self-vulcanizing tape and heat shrink tube were used. It has to be taken extreme care in the assembly of the cable glands for the optical fiber in order to prevent any leakage of water through this point and at the same time protect the delicate fibers from being damaged.

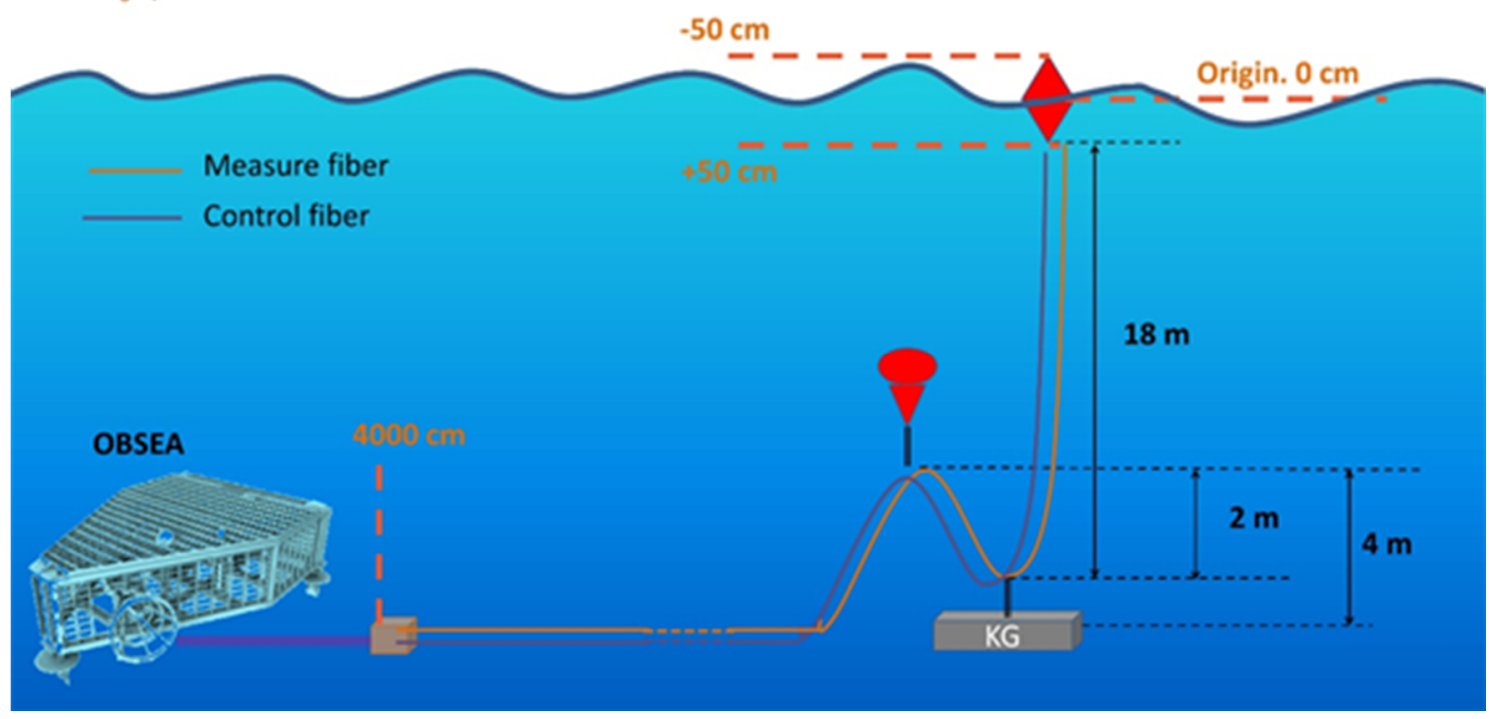

Figure 10 .Scheme of the onsite experiment

During the on site measurements several problems had to be faced. In January 2017, a storm destroyed all the experiment with irreparable damage in the fibers and severe damages in the surface buoy. Then a leakage was detected on the watertight cylinder that hosts the interrogator, with its possible corresponding irreversible damage. The holder device was repaired and the system had been collected data from June 2017 to September 2017.

\section{Sea water temperature measurements.}

At the time this paper was written, the measurements performed by optical fibers, CTDs were being analysed. Some conclusions came from this preliminary analysis.

Preliminary analysis of measurements taken along three months shows noisy measurements as is shown in Figure 11. Although the noise is lower than the resolution of the fiber optics, the movement of the fiber optics underwater the water could be an additional component of this noise since it was not observed during the calibration of the optical fibers. The fibers were calibrated in stirred liquid bath, with lower movement than in real conditions of use under the sea. Additional tests are needed to confirm the influence of the movement of the fiber optics on seawater temperature measurements. 


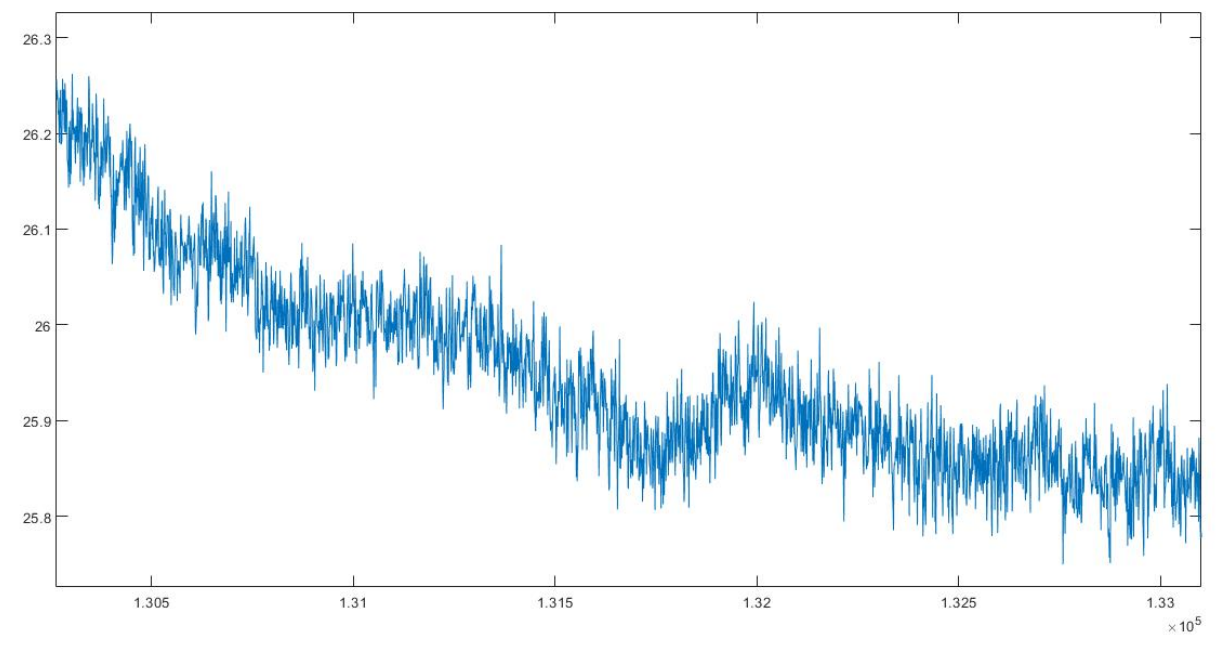

Figure 11. Noise of the seawater temperature measurements.

The data were statistically analysed in order to reduce the influence of the noise. The methodology used was the classification of the measurement data in sets of 20 samples. Each measurement was taken every 3 seconds and with an elapsed time of 1 minute between consecutive sets of 20 measurements

Temperature measurements taken by the fiber optic with 10 points of measurement over a specific time interval is shown in figure 12. This figure shows that the decrease in temperature with depth is lower than $3^{\circ} \mathrm{C}$ in the first 10 meters. Probably the high air temperatures along the summer and the fact that the time period corresponds to the end of the summer are fundamental factors to be considered in the low temperature variations. This explanation is in agreement with figure 13, where a difference of $6^{\circ} \mathrm{C}$ between the sea surface and $10 \mathrm{~m}$ depth is observed at the beginning of the summer. Even this agreement, further analysis and measurements in other seasons would be useful to confirm the explanation. 


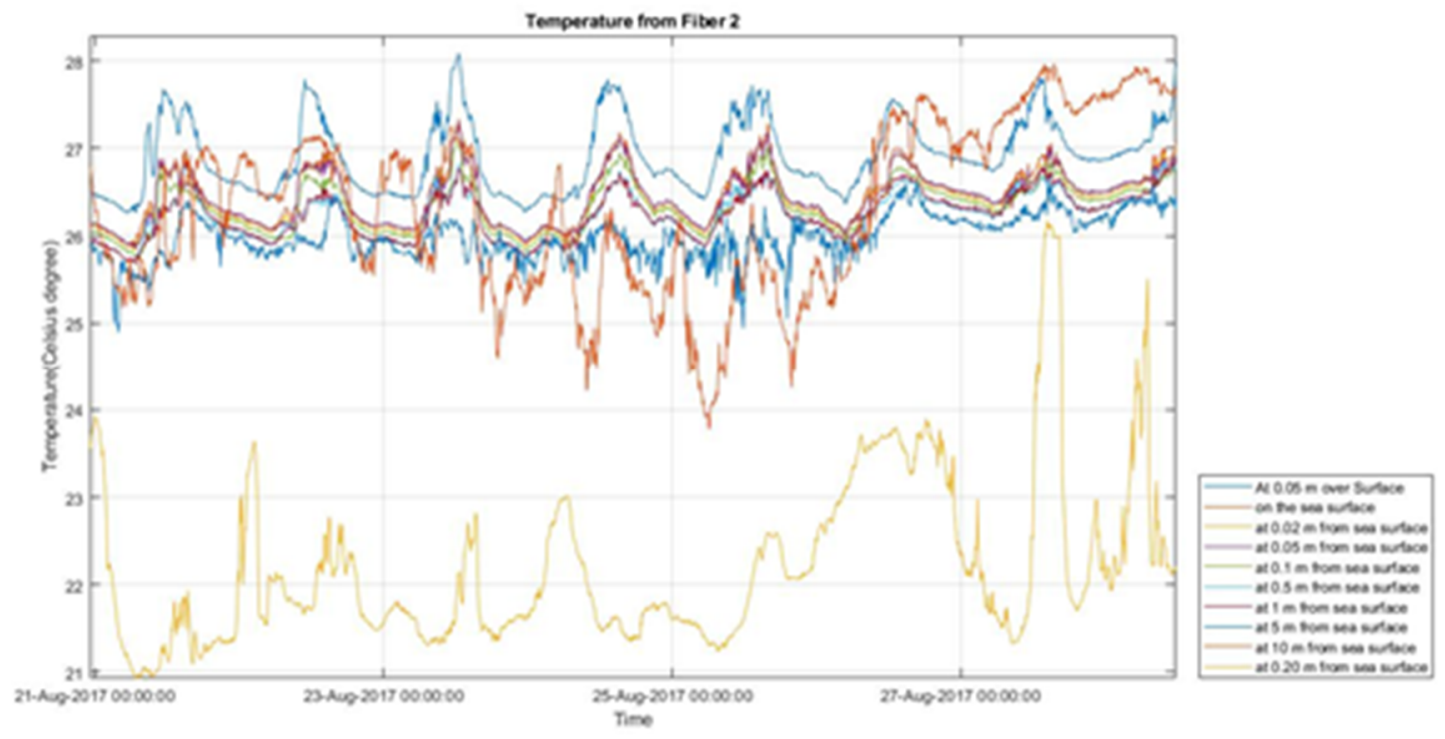

Figure 12. Seawater temperature profile in August.

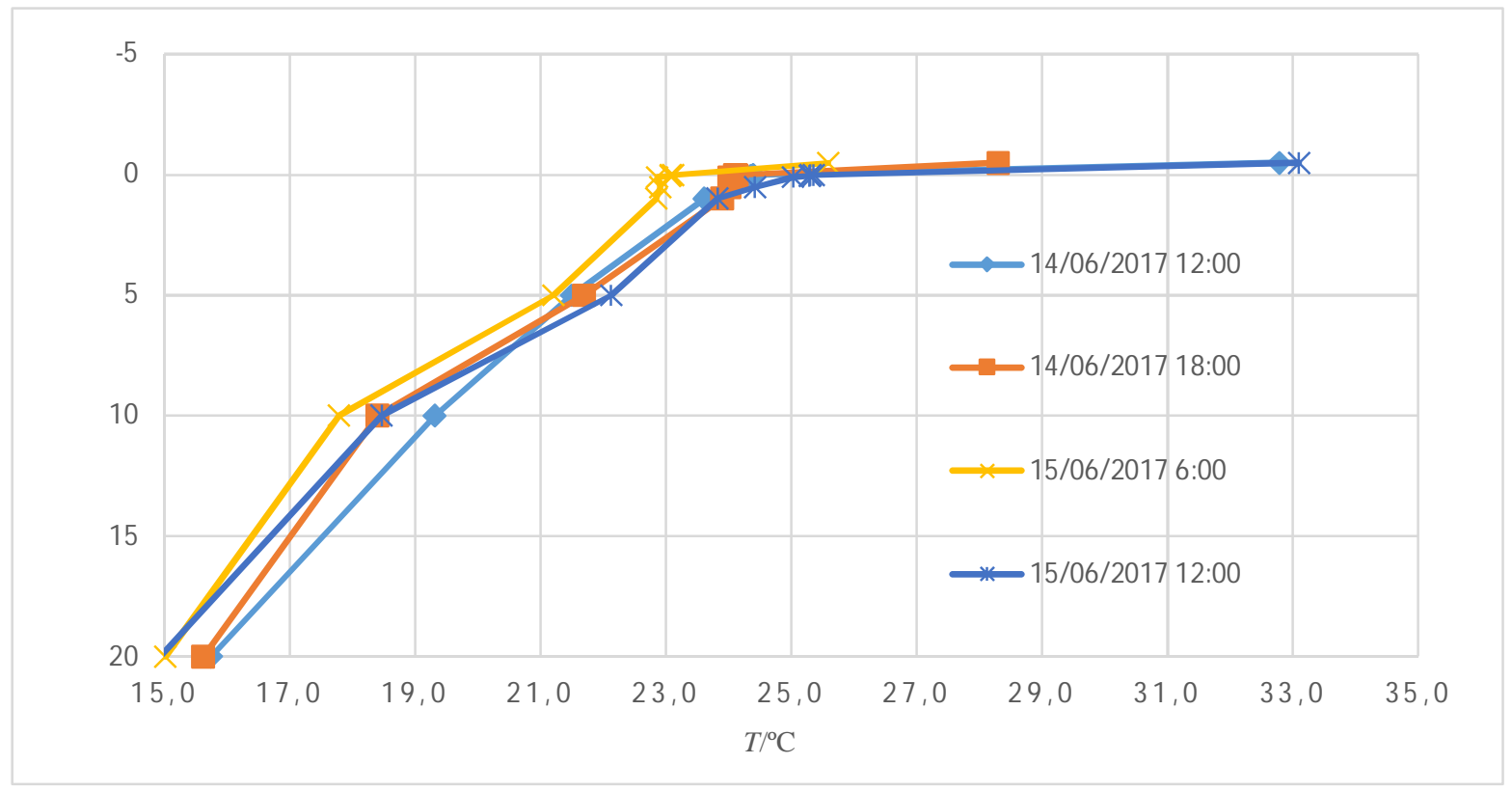

Figure 13. Sea water temperature profile in June

\section{Comparison between temperature measurements taken by fiber optics and the CTD}

The temperature measurements taken by fiber optics and by CTDs were compared. Preliminary analysis shows that the difference between both methods is lower than $0.1{ }^{\circ} \mathrm{C}$. Figures 14 and 15 show temperature measurements taken by both types of instruments in a specific time interval 


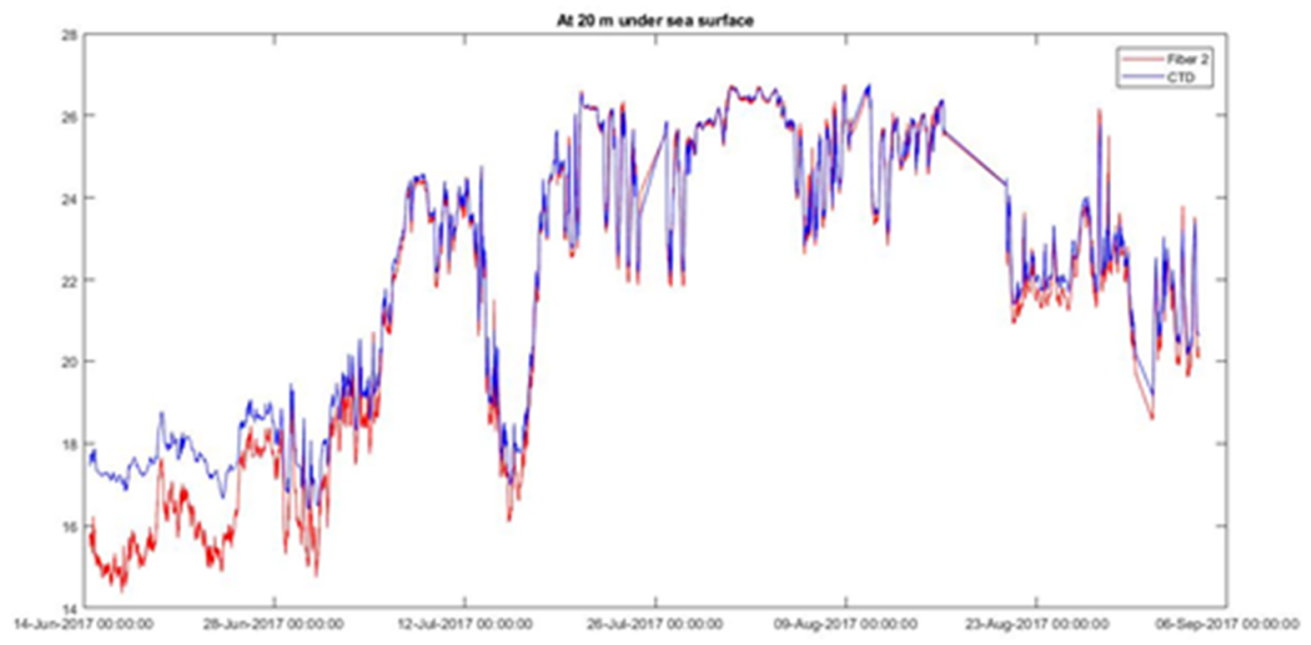

Figure 14. Values obtained by fiber optic and CTD, both at $20 \mathrm{~m}$ of depth.

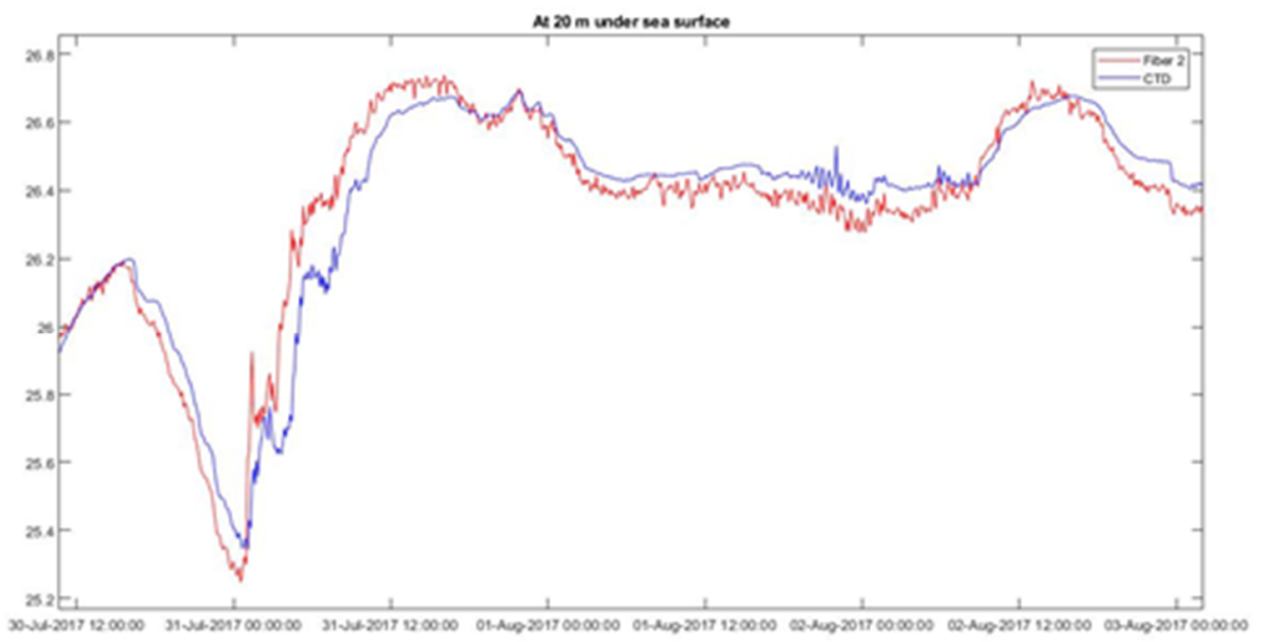

Figure 15. Zoom from figure 13

\section{Conclusions}

Optical systems based on optical fibers were designed, assembled and calibrated as optical instruments and as thermometers, with an expanded uncertainty of $80 \mathrm{mK}(k=2)$. The drift of almost all points of measurements are lower than the calibration uncertainty after three months of external exposure in marine environment

The CTD deployed at the Obsea underwater observatory was calibrated in a large calibration bath with an uncertainty of $5 \mathrm{mK}(k=2)$. The drift of the CTD seabird $37 \mathrm{SMD}$ after three months of use was analized, taking a value lower than $3 \mathrm{mK}$ in the calibration range $(0,30)^{\circ} \mathrm{C}$. 
A watertight system was designed and tested, hosting the interrogator (SM-125) of the fiber optics at the seabed and for three months.

The deployment of all the instrumentation associated with the experiment was performed and traceable sea temperature measurements were taken.

Additional tests are needed in order to check if the noise of the FBG readings are due to the movement of the fiber optics under the sea or if they are due to the change of marine currents temperature. Even thought, the fiber optics performed the measurement of traceable sea temperature profile and sea surface temperature during long periods of time.

Fiber Bragg Gratings (FBG), applied to seawater, was demonstrated as a feasible technique to continuously measure seawater temperature profile at open sea. Scientific feasibility was demonstrated through the study of the measurements drift and accuracy, although further investigations and works would be needed to improve the design and for hence, the uncertainties in temperature measurements. FBG could improve the problems of the actual systems based on winch or distributed thermistors in terms of power consumption and number of measurements points on the water column although more work it is necessary on such areas.

\section{Acknowledgments}

This work is supported by the project ENV58 MeteoMet2: "Metrology for essential climate variables" included inside the frame program EMRP. The EMRP is jointly funded by the EMRP participating countries within EURAMET and the European Union.This work was also developed by the EMRP Research Grant: ENV58-REG1, whose work was done in the project EMRP ENV58 MeteoMet2: "Metrology for essential climate variables"

\section{References}

[1] Marine Strategy Framework Directive, Directive 2008/56/EC of the European Parliament and of the Council. http://eur-lex.europa.eu/legalcontent/EN/TXT/?uri=CELEX:32008L0056 (accessed 13 December 2017).

[2] Jacopo Aguzzi, Antoni Mànuel, Fernando Condal, Jorge Guillén, Marc Nogueras, Joaquin Del Rio, Corrado Costa, Paolo Menesatti, Pere Puig, Francesc Sardà, Daniel Toma, Albert Palanques. "The new Seafloor Observatory (OBSEA) for remote and 
long-term coastal ecosystem monitoring”. Sensors vol. 11, Issue 6, 2011M.

[3] Salgado Díaz, Pedro; Martín López, Sonia; Carrasco Sanz, Ana y Corredera Guillén, Pedro, Método de calibración para interrogadores de redes de Bragg en fibra óptica, Óptica Pura y Aplicada, v. 45 (n. 3) pp. 361-368, 2012.

[4] GUM, "Guide to the Expression of Uncertainty in Measurement". 